\title{
SOME CONSIDERATION ON THE RATE OF INDUCED ALDOLASE SYNTHESIS IN AZOTOBACTER VINELANDII
}

\author{
SHIRO NAGAI, YOSHINORI NISHIZAWA, AND SHUICHI AIBA
}

The Institute of Applied Microbiology, University of Tokyo, Tokyo, Japan

(Received June 18, 1974)

\begin{abstract}
The rate of induced enzyme synthesis was formulated in general terms in line with a new account of the operon model by Yagil et al. who assumed originally two kinds of intracellular equilibria, one dealing with an effector (inducer) and free repressor, and the other, an operator and free repressor.

The rate of induced aldolase synthesis in Azotobacter vinelandii was examined during the unsteady state realized by a stepwise change in rotation speed of an impeller in a chemostat culture of this specific bacterium. Some of the observations, exhibiting incidentally the first-order response time, were in compliance with an analytical solution of the abovementioned formula, taking dissolved oxygen in the culture medium as an inducer. The first-order response in the rate of induced aldolase synthesis was given a new interpretation.
\end{abstract}

An obligate aerobe, Azotobacter vinelandii, is characterized by the fact that an increase of dissolved oxygen concentration in a culture medium from 0.1 to $4 \mathrm{ppm}$, for instance, results in a marked deterioration of growth yield, $Y_{X / S}(\mathrm{~g}$ cell $/ \mathrm{g}$ glucose) from 0.25 down to 0.03 . In connection with the observation that $Y_{X / S}$ values are affected appreciably by the dissolved oxygen concentration, aldolase activity was found to increase markedly with the increase in dissolved oxygen concentration. The enhanced activity of aldolase, which is responsible for the more marked evolution of carbon dioxide, could account for the deterioration of $Y_{X / S}$ values, since the principal metabolites other than the cells were carbon dioxide and water $(1,2)$.

Obviously, this observation provides a possibility that aldolase is induced by dissolved oxygen. The analysis of this induction, response-time in particular, remains to be studied from the operon model (3) which handles essentially the rate of induced and/or repressed enzyme synthesis, because the response-time 
either of the first- or of higher-order, omnipresent in the microbial world, needs to be reassessed from an aspect other than the engineering per se. This argument requires some elaboration below.

Although the operon theory has pointed out the mutual relationship among the repressor, co-repressor, and inducer to trigger and/or to suspend the functioning of an operator gene for transcribing mRNA in terms of induction and/or repression of intracellular enzyme, one may be confronted with the difficulty of how to apply this theory in the rate analysis of ad hoc enzyme induced and/or repressed by an environmental factor such as dissolved oxygen and/or glucose concentration in a culture medium.

Here, some works on enzyme induction and repression will be briefly reviewed to emphasize how the recent workers have attempted to shed light on their enzyme studies from an aspect of molecular biology, culminating, for instance, in the operon theory.

TeruI and his co-workers (4) studied induction of glucamylase and acid protease by Aspergillus niger, and $\alpha$-amylase by Bacillus subtilis in batch cultures. They introduced the term of mRNA in the analysis of kinetic behavior of these microorganisms. Briefly, the rate of change in specific activity of enzyme synthesis was equated to the term representing the specific growth rate, subtracting those relevant to "dilution effect" of the specific activity due to the growth rate, and first-order decomposition of mRNA.

By integrating the differential equation under various assumptions, they discussed the agreement and/or disagreement between the observation and calculation. Pertaining to application in the fermentation industry, they introduced initially mRNA in their enzyme studies. However, the substantial feature of the operon theory has not necessarily been fully appreciated in their work.

In this context, the recent work of IMANAKA et al. (5) on $\alpha$-galactosidase as induced and repressed by galactose and glucose in Monascus purpureus seems to have paid literally more attention to the theory. They proposed several differential equations for the induction and repression, and claimed to have had a favorable agreement between observation and calculation, though the procedure of how to find quite a number of rate constants and coefficients involved in these simultaneous equations for the numerical solution via a digital computer was left unclarified in their original paper.

YAGIL et al. (6), on the other hand, assumed equilibria among the effector (inducer or co-repressor), repressor, and operator intracellularly to have a clear view of the operon model. With respect to induction, for instance, they assumed two equilibria between inducer and repressor on one hand, and between operator and repressor on the other. By designating $\alpha$ to the fraction of a free operator in a microbial population, they presented the ratio of $\alpha /(1-\alpha)$ as a function of concentrations of inducer and repressor, incorporating the two equilibrium constants referred to above. 
Assuming further that the term $\alpha$ was accessible to actual observation of an enzyme induction and/or repression, they demonstrated their credibility by using data published by other workers on induction of $\beta$-galactosidase by isopropyl thiogalactoside in Escherichia coli and repression of alkaline phosphatase in $E$. coli by phosphate. Clearly, the new assessment by YAGIL et al. is considered to be candid, easy in access, and still to be most loyal to the original operon theory. The theoretical aspect of this work here will follow their new interpretation.

The purpose of the present work is to establish a kinetic model on the rate of induced enzyme synthesis and to check the applicability of this formula to the rate of aldolase induction in Azotobacter vinelandii when those cells are transferred from one steady state to another in a chemostat culture. Indeed, the unsteady state is a convenient means to manifest the rate of enzyme induction in this paper.

\section{THEORETICAL CONSIDERATION ON THE RATE OF INDUCED ENZYME SYNTHESIS}

YAGIL et al. (6) assumed the following two equilibria in microbial cells for a reassessment of the operon model.

$$
\begin{aligned}
& n I+R \stackrel{K_{1}}{\rightleftharpoons} R I_{n} \\
& O+R \stackrel{K_{2}}{\rightleftharpoons} O R
\end{aligned}
$$

where: $I=$ effector (inducer)

$R=$ unbound repressor

$O=$ free operator

$O R=$ operator bound by a repressor

$R I_{n}=$ repressor bound by $n$ moles of the inducer

$$
\begin{gathered}
K_{1}=\frac{[R][I]^{n}}{\left[R I_{n}\right]} \\
K_{2}=\frac{[O][R]}{[O R]}
\end{gathered}
$$

provided: $K_{1}$ and $K_{2}=$ equilibrium constants

Brackets [ ] signify concentration.

Eqs. (3) and (4) appearing below are concerned with mass balance for operator and repressor, respectively.

$$
\begin{gathered}
{\left[O_{t}\right]=[O]+[O R]} \\
{\left[R_{t}\right]=[R]+\left[R I_{n}\right]+[O R]}
\end{gathered}
$$

It is assumed that the third term on the right-hand side of Eq. (4) can be disregarded, compared with the other two terms, $[R]$ and $\left[R I_{n}\right]$,

$$
\left[R_{t}\right]=[R]+\left[R I_{n}\right]
$$


Cancelling out the terms $[R],\left[R I_{n}\right]$ and $[O R]$ from Eqs. $\left(1^{\prime}\right),\left(2^{\prime}\right),(3)$, and (4'), and solving $\alpha$ as defined by the ratio of $[O]$ to $\left[O_{t}\right]$,

$$
\alpha=\frac{[O]}{\left[O O_{t}\right]}=\frac{K_{1} K_{2}+K_{2}[I]^{n}}{K_{1} K_{2}+K_{2}[I]^{n}+K_{1}\left[R_{t}\right]}
$$

provided: $\left[O_{t}\right]=$ total concentration of the operator

$$
\left[R_{t}\right]=\text { total concentration of the repressor }
$$

The free operator concentration, $[O]$ in a microbial system is taken to be proportional to the specific change in the activity of induced enzyme, $\Delta E / \Delta X(6)$, where $\Delta E$ is the change in concentration of enzyme and $\Delta X$ is the change in concentration of cell mass.

Following the previous treatment by Yagil et al., the concentration of free operator, $[O]$, was assumed here to be taken as the ratio of a concentration change in relevant mRNA synthesized to that of total mRNA synthesized, i. e., $\Delta C_{m S} /$ $\Delta C_{m T}$. By the same token, the total concentration of operator, $\left[O_{t}\right]$, was represented by the maximum value of $\Delta C_{m S} / \Delta C_{m T}$, i. e., $\left(\Delta C_{m S} / \Delta C_{m T}\right)_{\max }$.

Then

$$
\alpha=\frac{[O]}{\left[O_{t}\right]}=\frac{\left(\frac{\Delta C_{m S}}{\Delta C_{m T}}\right)}{\left(\frac{\Delta C_{m S}}{\Delta C_{m T}}\right)_{\max }} \propto \frac{\left(\frac{\Delta C_{m S}}{\Delta X}\right)}{\left(\frac{\Delta C_{m S}}{\Delta X}\right)_{\max }} \propto \frac{\left(\frac{\Delta C_{m S}}{\Delta X^{\prime}}\right)}{\left(\frac{\Delta C_{m S}}{\Delta X^{\prime}}\right)_{\max }}
$$

where: $\quad X=$ cell mass concentration in $\mathrm{g}$ cell/liter

$X^{\prime}=$ soluble protein concentration in $\mathrm{g}$ protein/liter

$C_{m S}=$ concentration of relevant mRNA synthesized, mol/liter

$\mathrm{C}_{m T}=$ concentration of total $\mathrm{mRNA}$ synthesized, $\mathrm{mol} /$ liter

From Eq. (6),

where: $z_{\max }=\beta\left(\Delta C_{m S} / \Delta X^{\prime}\right)_{\max }$

$$
\alpha=\frac{1}{z_{\max }}\left(\frac{\Delta C_{m S}}{\Delta X^{\prime}}\right)
$$

$\beta=$ proportionality constant

Regarding the enzyme induction, a balance equation on the specific mRNA between synthesis via transcription of free operons and decomposition will be considered below.

$$
\frac{d C_{m S}}{d t}=\left(\frac{d C_{m S}}{d t}\right)_{S}+\left(\frac{d C_{m S}}{d t}\right)_{D}
$$

provided: $t=$ time, subscript: $S=$ synthesis, $D=$ decomposition

Assuming the decomposition rate of the specific mRNA as a first-order reaction $(4,5)$,

$$
\left(\frac{d C_{m S}}{d t}\right)_{D}=-k_{2} C_{m S}
$$


provided: $\quad k_{2}=$ decomposition rate constant, $\mathrm{hr}^{-1}$

Eqs. (7) to (9) are rearranged to give the following equation, if the difference symbol, $\Delta$, in Eq. (7) can be transformed to the differential, $d$.

$$
\frac{d C_{m S}}{d t}=z_{\max } \alpha\left(\frac{d X^{\prime}}{d t}\right)-k_{2} C_{m S}
$$

Now, the rate of enzyme synthesis in a microbial system which is proliferating is assumed to be proportional to the concentration of the specific mRNA in the system, subtracted by the first-order decomposition rate of the enzyme $(4,5)$.

$$
\frac{d E}{d t}=k_{3} C_{m S}-k_{4} E
$$

where: $\quad E=$ activity of specific enzyme, units $/ \mathrm{ml}$

$k_{3}=$ rate constant for enzyme synthesis, $\mathrm{hr}^{-1}$

$k_{4}=$ rate constant for enzyme decomposition, $\mathrm{hr}^{-1}$

Differentiating further Eq. (11) with time, $t$,

$$
\frac{d^{2} E}{d t^{2}}=k_{3} \frac{d C_{m S}}{d t}-k_{4} \frac{d E}{d t}
$$

Cancelling out $d C_{m S} / d t$ and $C_{m S}$ from Eqs. (10), (11), and (11'),

$$
\frac{d^{2} E}{d t^{2}}+\left(k_{2}+k_{4}\right) \frac{d E}{d t}+k_{2} k_{4} E=k_{3} z_{\max } \alpha \frac{d X^{\prime}}{d t}
$$

Here, the term $\varepsilon$, which means the specific rate of enzyme synthesis, is introduced:

$$
\varepsilon=\frac{1}{X^{\prime}} \frac{d E}{d t}=\frac{1}{X^{\prime}} \frac{d\left(N X^{\prime}\right)}{d t}=\frac{d N}{d t}+\mu N
$$

where: $\quad N=E / X^{\prime}=$ specific activity of enzyme, units $/ \mathrm{mg}$ protein

$\mu=\left(1 / X^{\prime}\right)\left(d X^{\prime} / d t\right)=$ specific growth rate, $\mathrm{hr}^{-1}$

$\varepsilon=$ specific rate of enzyme synthesis, units $/ \mathrm{mg}$ protein $/ \mathrm{hr}$

From Eqs. (12) and (13),

$$
\frac{d \varepsilon}{d t}+\left(k_{2}+k_{4}+\mu\right) \varepsilon+k_{2} k_{4} N=k_{3} z_{\max } \alpha \mu
$$

If the value of $K_{4}$ in Eq. (11) is assumed to equal zero, Eq. (14) is reduced to:

$$
\frac{d \varepsilon}{d t}+\left(k_{2}+\mu\right) \varepsilon=k_{2} z_{\max } \alpha \mu
$$

Taking $n=1$ and $[I] \gg K_{1}$ in Eq. (5), the equation is reduced to:

$$
\alpha=\frac{C}{K+C}
$$

where: $K=K_{1}\left[R_{t}\right] / K_{2}$

$C=[I], i . e .$, the concentration of inducer here pertaining to aldolase in $A$. 
vinelandii is taken as the concentration of dissolved oxygen in the medium

From Eqs. (15) and (16),

$$
\frac{d \varepsilon}{d t}+\left(k_{2}+\mu\right) \varepsilon=\frac{k_{2} z_{\max } C}{K+C}
$$

Regarding unsteady state realized by disruption of a chemostat culture of the specific microbe, Azotobacter vinelandii, the values of $\varepsilon, \mu$, and $C$ are due to change with time. If and only if the values of $\mu$ and $C$ remain fortuitously unchanged under specific experimental conditions, an analytical solution to Eq. (17) is:

$$
\varepsilon=J \frac{C \mu}{K+C}+\left(\varepsilon_{0}-J \frac{C \mu}{K+C}\right) \exp \left\{-\left(\mu+k_{2}\right) t\right\}
$$

where: $J=\frac{k_{3} z_{\max }}{\mu+k_{2}}$

$$
\varepsilon_{0}=\text { value of } \varepsilon \text { at } t=0 \text { when the unsteady period is initiated }
$$

Generally, when the values of $\varepsilon, \mu$, and $C$ in Eq. (18) are subjected to change with time, a numerical solution to the equation by a digital computer to obtain a more comprehensive check on the time-delay in the rate of induced aldolase synthesis will be discussed separately.

Substituting Eq. (18) into the left-hand side of Eq. (13), and solving another linear differential equation with respect to $N$,

$$
\begin{aligned}
& N=\frac{J C}{K+C}+\left\{N_{0}+\frac{\varepsilon_{0}}{k_{2}}-\frac{J C \mu}{k_{2}(K+C)}-\frac{J C}{K+C}\right\} \exp (-\mu t) \\
& -\left\{\frac{\varepsilon_{0}}{k_{2}}-\frac{J C \mu}{k_{2}(K+C)}\right\} \exp \left\{-\left(\mu+k_{2}\right) t\right\}
\end{aligned}
$$

provided: $\quad N_{0}=$ value of $N$ at $t=0$

If the value of $t$ approaches infinity (steady state) in Eq. (20),

$$
N=\frac{J C}{K+C}
$$

\section{MATERIALS AND METHODS}

Strain and culture condition. The strain used throughout was Azotobacter vinelandii IAM 1078 (ATCC 9046). Compositions of the culture medium used were, in $\mathrm{g} /$ liter, glucose, 8.0; $\mathrm{KH}_{2} \mathrm{PO}_{4}, 0.2 ; \mathrm{K}_{2} \mathrm{HPO}_{4}, 0.8 ; \mathrm{MgSO}_{4} \cdot 7 \mathrm{H}_{2} \mathrm{O}, 0.2$; $\mathrm{NaCl}, 0.2$; sodium citrate, $0.05 ; \mathrm{FeSO}_{4} \cdot 7 \mathrm{H}_{2} \mathrm{O}, 0.005 ; \mathrm{Fe}_{2}\left(\mathrm{SO}_{4}\right)_{3} \cdot 3 \mathrm{H}_{2} \mathrm{O}, 0.005$; $\mathrm{Na}_{2} \mathrm{MoO}_{4} \cdot 2 \mathrm{H}_{2} \mathrm{O}, 0.001 ; \mathrm{CaCl}_{2}, 0.01 ; \mathrm{pH}=7.0$ to 7.2 .

The cell suspension ( $200 \mathrm{ml}$ in volume, pre-cultured at $30^{\circ}$ for $24 \mathrm{hr}$ ) was inoculated into a 5-liter and fully baffled fermentor (working volume $=2.2$ liter), equipped centrally with a six flat bladed turbine and a multi-hole sparger. When the cell mass concentration attained an appropriate level in a batch culture, a fresh 
and sterile medium was charged from a reservoir into the vessel by a peristaltic pump (Taiyo Kagaku Kogyo Co., Ltd., Tokyo) with a fixed rate that the dilution rate, $D$, was about $0.2 \mathrm{hr}^{-1}$.

The medium from the fermentor was discharged at the same rate from an overflow pipe fixed inside the vessel. Aeration rate was $0.6 \mathrm{vvm}$ and continuous cultivation was also at $30^{\circ}$. The rotation speed of the impeller extended from 400 to $600 \mathrm{rpm}$ in each chemostat to realize various concentrations of dissolved oxygen in the medium. The steady state was assumed if the cell mass concentration remained nearly unchanged for at least $20 \mathrm{hr}$.

For a quick estimation of the cell concentration, the value of optical density at $610 \mathrm{~nm}$ was employed. As will be seen from the experimental data appearing later, either glucose or dissolved oxygen might have been limiting in each chemostat culture.

Disruption of chemostat culture. Without suspending the charge of a fresh medium into the fermentor vessel, the rotation speed of the impeller was exclusively changed (increased or decreased) stepwise, leaving the aeration rate and other cultivation conditions intact, when the unsteady state was required. During the unsteady state, various measurements with respect to aldolase activity, dissolved oxygen concentration, and so on were conducted from time to time.

Actually, the change in rotation speed of the impeller was to attain rapidly a new level of dissolved oxygen different from that in the preceding chemostat culture. The reason for having adopted dissolved oxygen concentration to realize the unsteady state stems from the previous study (1) that aldolase was most sensitive to the concentration of dissolved oxygen in the culture medium.

Analytical procedures. Cell mass: Broth samples from the fermentor vessel were processed separately and in advance with a mixture of acetone and Dry Ice to obtain a powdered cell material (water content, 15\%) (1). This determination yielded an empirical correlation between the cell mass concentration and the reading of optical density at $610 \mathrm{~nm}$. This correlation permitted the quick assessment of cell mass concentration as was referred to earlier.

Glucose: The concentration of glucose in the culture medium was measured either by the Somogyi or the Glucostat method.

Dissolved oxygen: Membrane-covered electrodes (Toshiba-Beckman Type 777 and Denki Kagaku Keiki Co., Tokyo) were used to measure the concentration of dissolved oxygen in the broth.

Soluble protein: The method of GoRnall et al. (7) was employed; the reading was converted to a value based on that of bovine albumin.

Aldolase: Before the measurement of cellular activity of aldolase, a crude extract of the enzyme was prepared by centrifugation of the sampled broth $(50 \mathrm{ml})$ $\left(5^{\circ}, 8,000 \times g\right.$ for $\left.5 \mathrm{~min}\right)$, followed by rising the sediment and its resuspension in a phosphate buffer solution first and, second, by treatment of the resuspension with a sonic disintegrator (Tominaga Works Ltd., Tokyo, Model UR-1500 P, 20 kHz, 
$30 \mathrm{sec}$ ), followed again by another centrifugation $\left(5^{\circ}, 25,000 \times g\right.$ for $\left.5 \mathrm{~min}\right)$ to secure a supernatant liquid.

A UV-kit for aldolase (C. F. Boehringer Mannheim $\mathrm{GmbH}$, Biochemica, Germany) was used to assess the activity of aldolase in the crude preparation. Briefly, the rate of decrease in $\mathrm{NADH}_{2}$ absorption spectrum at $340 \mathrm{~nm}$ was observed with an automatic recording-type spectrophotometer (Hitachi Ltd., Tokyo, Model 101). The molecular extinction of $\mathrm{NADH}_{2}$ was taken as $6.22 \times 10^{6} \mathrm{~cm}^{2} / \mathrm{mol}$. The rate of decrease in $\mathrm{NADH}_{2}$ in $\mu \mathrm{mol} / \mathrm{min}$ was designated as one unit of aldolase activity (1).

\section{RESULTS AND DISCUSSION}

Experimental data on the specific activity of aldolase, $N$, units/mg protein, $v s$. dissolved oxygen concentration, $C, \mathrm{mmole} / \mathrm{ml}$, either in glucose-limited or in oxygenlimited chemostat culture of Azotobacter vinelandii are shown in Fig. 1. Obviously, a hyperbolic pattern of $N$ increasing with the increase in $C$ values is demonstrated ( $c f$. the solid curve assumed).

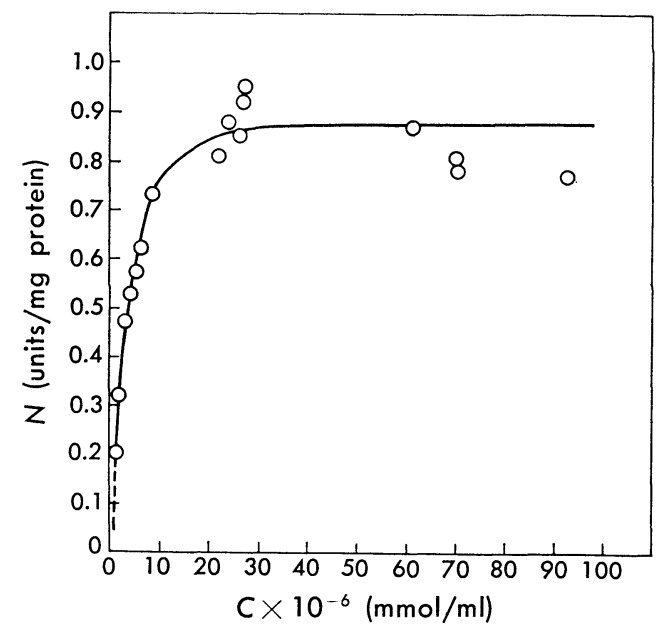

Fig. 1. Effect of dissolved oxygen concentration on specific activity of aldolase in glucose- or oxygen-limited chemostat culture of Azotobacter vinelandii. $\quad D \fallingdotseq 0.2 \mathrm{hr}^{-1}$.

However, these experimental data in Fig. 1 are remarkably different from the trend observed previously on aldolase activity as affected by dissolved oxygen concentration. The previous trend (1) between aldolase activity expressed in $\mu \mathrm{mol} / \mathrm{mg}$ protein/min was linear against the concentration of dissolved oxygen which covered nearly the whole range of $C$ values on the abscissa of Fig. 1. The 
only difference between the current and the previous work was that the dilution rate in the present work was kept nearly unchanged at $D=0.2 \mathrm{hr}^{-1}$, the value of $C$ being manipulated by the rotation speed of the impeller, whereas the value of $D$ ranged from 0.1 to $0.3 \mathrm{hr}^{-1}$, yielding necessarily the change in $C$ values in each chemostat of the previous study (1).

The reason for this discrepancy may lie in Eqs. (19) and (21), i.e.,

$$
N=k_{3} z_{\max }\left(\frac{1}{\mu+k_{2}}\right)\left(\frac{C}{K+C}\right)
$$

It is clear from Eq. (22) that $N$ is proportional to $C /\left(\mu+k_{2}\right)(K+C)$. Although the values of $k_{2}$ and $K$ in the previous work are beyond assessment, the value of $\left(\mu+k_{2}\right)(K+C)$ might have been nearly unchanged regardless of the change in $C$ values. Actually, the increase of $\mu(=D)$ decreased the value of $C$ and vice versa. The complementary feature of $\mu(=D)$ and $C$ appearing in the two parentheses, $\left(\mu+k_{2}\right)$ and $(K+C)$, might have minimized the variation of $\left(\mu+k_{2}\right)(K+C)$ against $C$.

In order to estimate the values of $J$ and $K$ in Eq. (21), the data points in Fig. 1 were subjected to the double reciprocal plot as shown in Fig. 2. The solid line assumed through the data (open circle) in Fig. 2 gave:

$$
\left.\begin{array}{rl}
J & =0.9 \text { units } / \mathrm{mg} \text { protein } \\
K & =2.75 \times 10^{-6} \mathrm{M}
\end{array}\right\}
$$

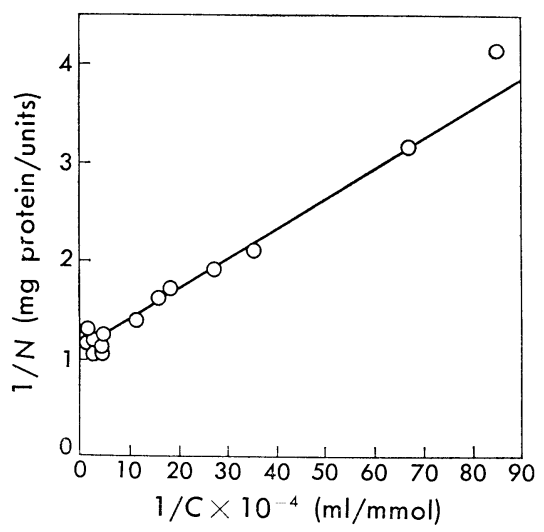

Fig. 2. Double reciprocal plot of specific activity of aldolase against dissolved oxygen concentration.

An example of unsteady pattern of $N$ is shown in Fig. 3; this graph points out that an oxygen-limited chemostat culture of Azotobacter vinelandii had been realized before the disturbance of dissolved oxygen concentration was imposed at $t=0$ by changing the rotation speed of the impeller stepwise from 400 to $525 \mathrm{rpm}$, and keeping the dilution rate and aeration rate unaltered. 
In this example (for $t>0$ ), the values of $C$ and $X$ remained nearly unchanged. This latter fact of constant value of $X$ implies that the specific growth rate of $A$. vinelandii remained unaffected $\left(\mu=0.2 \mathrm{hr}^{-1}\right)$ in spite of this disturbance. However, the value of $S$ decreased gradually such that the "oxygen-limited" chemostat was transferred to another chemostat, though the ultimate nature at another steady state is beyond discussion from Fig. 3.

The specific activity of aldolase, $N$, on the other hand, responded appreciably to the change in dissolved oxygen concentration, $C$, that the growth yield, $Y_{X / S}$, of $A$. vinelandii was lowered in view of the fact that the values of $X$ remained unaltered despite the apparent increase in glucose consumption during the unsteady period. The response of $N$ to the enhancement of $C$ in Fig. 3 was, therefore, in agreement with the previous finding (l) that the value of $N$ increased with the increase of $C$, resulting in the deterioration of $Y_{X / S}$.

Contrary to a prompt increase in dissolved oxygen concentration, $C$ for $t>0$, the response of specific activity of aldolase, $N$, clearly exhibits a delay, resembling the first-order delay. This, in turn, suggests that a calculation of $N$ as a function of time, $t$, be attempted by Eq. (20), because the value of $X, C$, and $\mu$ with respect to the period for $t>0$ in Fig. 3 could be assumed to be respectively constant, and

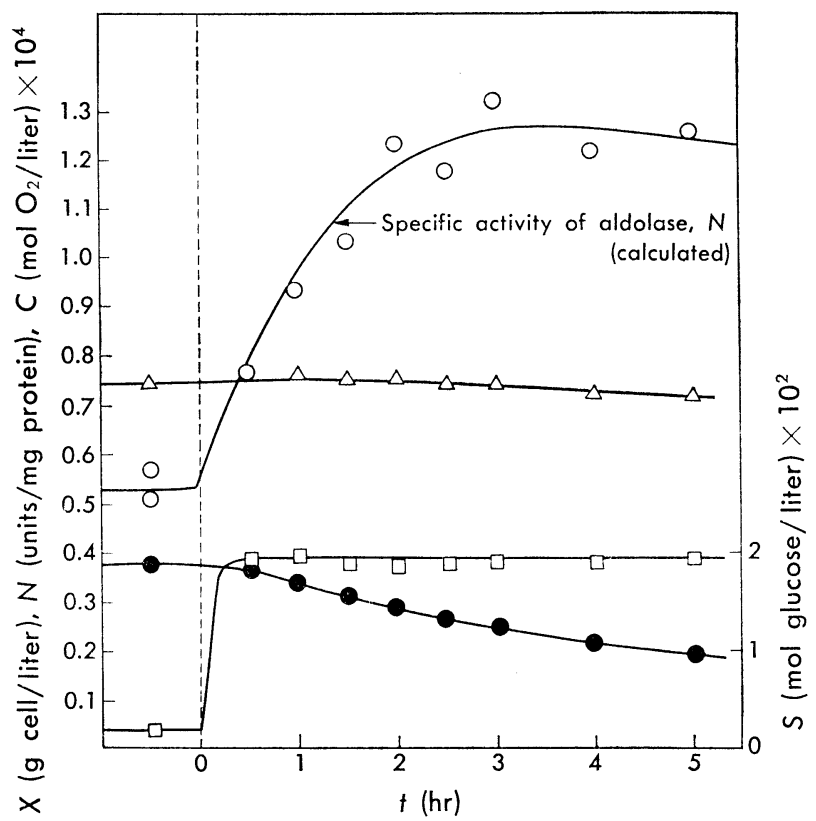

Fig. 3. Specific activity of aldolase $(N)$, glucose concentration $(S)$, and cell mass concentration $(X)$ as varying with time in unsteady state caused by a change in dissolved oxygen concentration $(C)$ stepwise from 3.6 to $38 \mu \mathrm{M}$.

$\bigcirc: N$, units/mg protein, $\triangle: X$, g cell/liter, $\bullet: S$, mol/liter, $\square: C$, mol/liter. 
then, the analytical solution of Eq. (20) could be consulted.

The calculation of $N$ with Eq. (20) was made by substituting the following values into the equation.

$$
\begin{aligned}
J & =0.9 \text { units/mg protein } \\
K & =2.75 \mu \mathrm{M} \\
C & =38 \mu \mathrm{M} \\
\mu & =0.2 \mathrm{hr}^{-1} \\
\varepsilon_{0} & =\left(\frac{d N}{d t}+N \mu\right)_{t=0} \quad \text { (cf. Eq. (23)) } \\
& =0.6+0.53 \times 0.2=0.71 \text { units/mg protein } / \mathrm{hr} \\
k_{2} & =0.31 \mathrm{hr}^{-1}
\end{aligned}
$$

The use of $k_{2}=0.31 \mathrm{hr}^{-1}$ had no particular significance; this was so estimated that the calculation could represent fairly well the observation of $N$ in Fig. 3, to say nothing of the fact that $J$ and $K$ values were assessed from the chemostat culture, far from the actual transient period. This kind of agreement does not substantiate altogether the assumptions underlying the analysis of the rate of induced enzyme synthesis. In spite of this critical argument, the response of aldolase synthesis induced by dissolved oxygen concentration, as shown apparently here by the first-

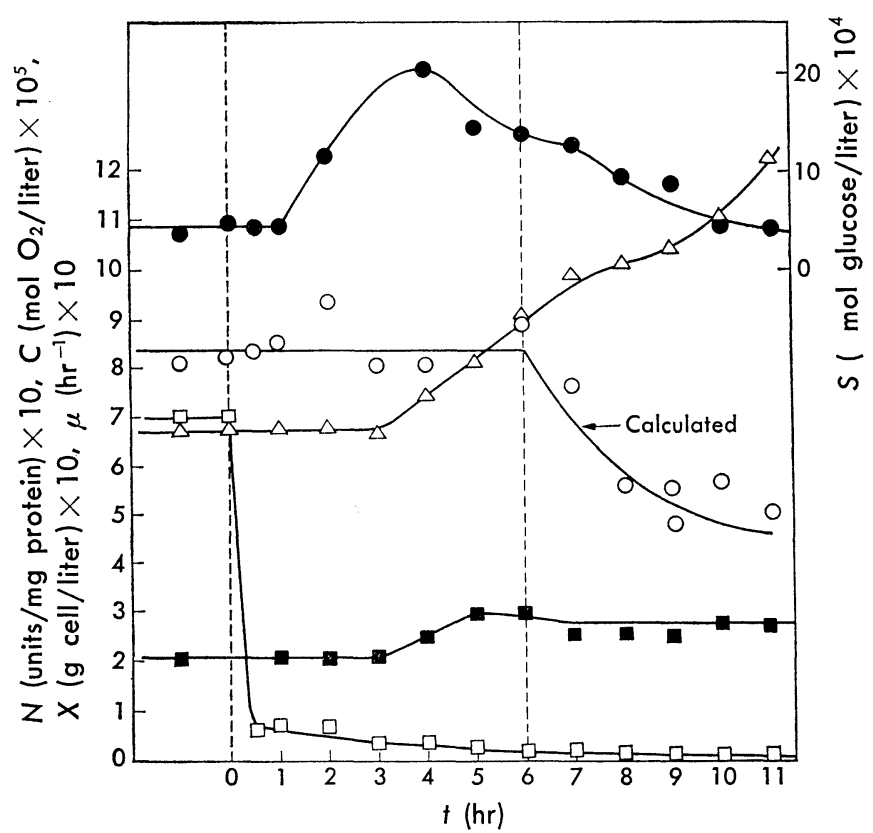

Fig. 4. Specific activity of aldolase $(N)$, glucose concentration $(S)$, and cell mass concentration $(X)$ as varying with time in unsteady state caused by a change in dissolved oxygen concentration $(C)$ stepwise from 70 to $2 \mu \mathrm{M}$.

$\bigcirc: N$, units/mg protein, $\triangle: X, \mathrm{~g}$ cell/liter, $\bullet: S, \mathrm{~mol} /$ liter, $\square: C, \mathrm{~mol} / \mathrm{liter}, \mathbf{\square}: \mu, \mathrm{hr}^{-1}$. 
order time-delay, has been interpreted most likely from another aspect based essentially on the operon theory.

Another example of transient behavior of this bacterial aldolase is shown in Fig. 4. In this experiment, rotation speed of the impeller decreased from 600 to $500 \mathrm{rpm}$ at $t=0$ in this graph. Contrary to Fig. 3, it is noted from Fig. 4 that the values of $C$ dropped sharply to lower levels and those of $S$ were elevated after a short lapse of time in this period of $t>0$. It is also remarked from Fig. 4 that the concentration of cell mass, $X$, did not remain unchanged, while $N$ was kept unaltered in the level for a considerable period of time (about $6 \mathrm{hr}$ ) after the initiation of this change in rotation speed of the impeller.

Incidentally, when $t>6 \mathrm{hr}$ in this example, the decrease of $C$ to an extent of $C=2 \mu \mathrm{M}$ did trigger the decrease of $N$ values contrary to the trend in Fig. 3 . The response of induced aldolase synthesis rate (degradation here) (open circles in Fig. 4) also points out the first-order delay as could be accounted for by Eq. (20), if and only if the values of $\mu$ and $C$ could be assumed to be constant.

In order to check whether or not the values of $\mu$ for $t>6 \mathrm{hr}$ could be regarded constant, the following mass balance equation was employed.

$$
\mu=D+\frac{1}{X} \frac{d X}{d t}
$$

where: $\quad D=0.21 \mathrm{hr}^{-1}$

By using appropriately the data points of $X$ (triangles), the second term in the right-hand side of Eq. (24) was estimated to obtain $\mu$ values. The result of this assessment is shown in Fig. 4 by solid squares.

It is clear from Fig. 4 that the values of $\mu$ and $C$ were also incidentally and fairly constant after $t=6$ in this graph. Then, transferring the origin of $t^{\prime}$ from $t=0$ to $t=6$, and substituting the following values into Eq. (20), the value of $N$ responding to the value of $C$ was calculated as shown by the solid curve through open circles for $t^{\prime}>0$.

$$
\begin{aligned}
J & =0.9 \text { units/mg protein } \\
K & =2.75 \mu \mathrm{M} \\
C & =2 \mu \mathrm{M} \\
\mu & =0.28 \mathrm{hr}^{-1} \\
\varepsilon_{0} & =\left(\frac{d N}{d t}+N \mu\right)_{t^{\prime}=0} \quad(c f . \text { Eq. }(23)) \\
& =-0.18+0.58 \times 0.28=-0.058 \text { units/mg protein } / \mathrm{hr} \\
k_{2} & =0.23 \mathrm{hr}^{-1}
\end{aligned}
$$

Here again, the agreement between calculation and observation as noted from Fig. 4 demonstrates the possibility that the first-order time delay in the response of induced rate of aldolase synthesis could be envisaged from the theoretical approach underlying Eq. (20), though the arbitrary selection of $k_{2}=0.23 \mathrm{hr}^{-1}$ also in this case was accompanied by no special implication per se. 


\section{REFERENCES}

1) S. Nagai, Y. Nishizawa, M. Onodera, and S. Aiba, J. Gen. Microbiol., 66, 197 (1971).

2) S. NAgAi and S. AibA, In Fermentation Technology Today, ed. by G. TerUi, Soc. Ferment. Technol., Japan (1972), p. 143.

3) F. JACOB and J. Monod, J. Mol. Biol., 3, 318 (1961).

4) A. Shinmyo, M. Okazaki, and G. Terui, In Fermentation Advances, ed. by D. Pearlman, Academic Press, New York (1969), p. 337.

5) T. Imanaka, T. Kaieda, K. Sato, and H. Taguchi, J. Ferment. Technol., 50, 633 (1972).

6) G. Yagil and E. Yagil, Biophys. J., 11, 11 (1971).

7) A. G. Gornall, C. S. Bardawill, and M. M. David, J. Biol. Chem., 177, 751 (1949). 